\title{
Resultados clínicos del tratamiento de pacientes con hernia inguinal unilateral mediante cirugía ambulatoria
}

\author{
Clinical outcomes of ambulatory surgical treatment of unilateral inguinal hernia \\ Roberto Ernesto Vigil-Guerrero $^{1, a, b}$ (D) , Mario Augusto Del Castillo Yrigoyen ${ }^{2, c}$ \\ RESUMEN
}

Objetivo: Describir los resultados clínicos del tratamiento de pacientes con hernia inguinal unilateral (HIU) realizados en una unidad de cirugía mayor ambulatoria de un hospital de nivel III. Material y métodos: Se revisaron las historias clínicas de los pacientes operados de HIU en la Unidad de Cirugía Mayor ambulatoria (UCMA) del Hospital Cayetano Heredia (HCH) entre julio del 2013 y junio del 2016. Resultados: El número final de pacientes incluidos fue 154. La edad promedio fue 43 años; $101(65,6 \%)$ fueron varones; 104 (67,5\%) tenían riesgo quirúrgico ASA I; $12(7,8 \%)$ tenían enfermedades asociadas; $94(61,0 \%)$ se operaron con la técnica abierta, y $60(39,0 \%)$ por la vía laparoscópica. La cirugía laparoscópica fue usada con más frecuencia en las mujeres ( $\mathrm{p}=0.037)$. El tiempo operatorio fue mayor en la cirugía laparoscópica $(\mathrm{p}=0.033)$. No hubo diferencia significativa con respecto a la edad, riesgo quirúrgico, complicaciones anestésicas y complicaciones quirúrgicas entre pacientes operados con cirugía abierta y con cirugía laparoscópica. En total 34 (22\%) pacientes no salieron de alta el mismo día de la cirugía: Ocho $(5,2 \%)$ pacientes por presentar complicaciones y $26(16,8 \%)$ pacientes por razones administrativas/ sociales. Conclusión: La cirugía ambulatoria de HIU en adultos realizada por la técnica abierta o laparoscópica, es un procedimiento seguro que permite el alta del paciente el mismo día de la cirugía.

PALABRAS CLAVE: Hernia inguinal, procedimientos quirúrgicos ambulatorios, laparoscopía. (Fuente: DeCS BIREME).

\section{SUMMARY}

Objective: To describe the clinical outcomes of patients with unilateral inguinal hernia (UIH) treated in an ambulatory surgical unit (ASU) of a level III hospital. Methods: The clinical charts of patients treated at the ASU of Cayetano Heredia Hospital from July 2013 to June 2016 were reviewed. Results: 154 patients were included, mean age was 43 years; 101 (65.6\%) were males; 104 (67.5\%) had ASA I risk; 12 (7.8\%) had underlying diseases; 94 (61.0\%) undergone the open technique and $60(39.0 \%)$ undergone laparoscopy. Laparoscopy was most frequently applied in women $(\mathrm{p}=0.037)$. Surgical time was higher in laparoscopic procedures $(\mathrm{p}=0.033)$. No significant differences

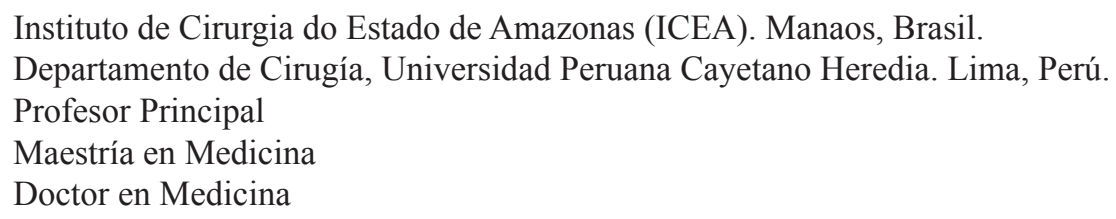


were observed in variables such as age, surgical risk, anesthetic and surgical complications between open and laparoscopic procedures. Thirty-four (22\%) patients were not discharged the day of the procedure: $8(5.2 \%)$ presented complications and $26(16.8 \%)$ had administrative or social reasons. Conclusion: Ambulatory surgery of UIH in adults either by open or laparoscopic procedures is safe and allows discharging patients the day of the procedure.

KEYWORDS: Groin hernia, laparoscopy, ambulatory surgery procedures, inguinal herniorrhaphy. (Source: MeSH NLM).

\section{INTRODUCCIÓN}

En los últimos años el aumento de pacientes que asisten a la consulta de cirugía general del Hospital Cayetano Heredia $(\mathrm{HCH})$ con el diagnóstico de hernia inguinal hizo que cambien las estrategias para atender la demanda insatisfecha, reducir los tiempos prolongados de espera para una cirugía y el tiempo de hospitalización ${ }^{(1)}$. En este escenario, en el 2013, se aprobó la directiva que permitió el funcionamiento de la Unidad de Cirugía Mayor Ambulatoria (UCMA) en nuestro hospital, con la finalidad que pacientes quirúrgicos, entre los que se encontraban aquellos con diagnóstico de hernia inguinal y que cumplían con los requisitos de esta directiva, podrían operarse en forma ambulatoria ${ }^{(2)}$.

La cirugía de la hernia en programas de cirugía ambulatoria, comparados con los sistemas de hospitalización, demostraron la misma seguridad y efectividad, alta aceptación por los pacientes y menores $\operatorname{costos}{ }^{(3)}$. La técnica operatoria considerada como referencia de la reparación de hernia inguinal es la hernioplastía de Lichtenstein (cirugía sin tensión con el uso de malla). Pocos discuten la reproducibilidad del procedimiento, sus ventajas en términos de aprendizaje y su alta rentabilidad en términos de costos. Tampoco se discuten sus resultados satisfactorios acortando el periodo de recuperación, disminuyendo las molestias post operatorias inmediatas, y reduciendo al mínimo la estancia hospitalaria ${ }^{(4)}$.

Con los adelantos de la anestesia general, uso de mascarilla laríngea, los nuevos fármacos y la monitorización con nuevas tecnologías, las unidades de cirugía mayor ambulatoria han aceptado la cirugía laparoscópica como una alternativa importante en la cirugía de hernia ${ }^{(5)}$. La hernioplastía inguinal laparoscópica obtiene beneficios adicionales como es la rápida recuperación física y funcional, menor dolor en el postoperatorio, menor consumo de analgésicos, menor tasa de dolor inguinal crónico y con índices de recurrencia semejantes a la cirugía convencional ${ }^{(6)}$.

La posibilidad de cirugía ambulatoria comienza a ser considerada para todos los pacientes, independientemente de la técnica de reparación y el tipo de anestesia, donde el protocolo asegure una buena selección de pacientes, consulta y orientación pre anestésica además de un respaldo familiar ${ }^{(7)}$.

El tiempo de espera de pacientes con hernia inguinal es prolongada, por lo que la UCMA es una opción importante para operar pacientes con esta patología sin necesidad de hospitalizarlos.

El objetivo del estudio fue describir los resultados clínicos del tratamiento quirúrgico ambulatorio de pacientes con hernia inguinal unilateral (HIU) realizada mediante la técnica de Lichtenstein y la técnica laparoscópica.

\section{MATERIAL Y MÉTODOS}

Estudio descriptivo, transversal, retrospectivo. Se revisaron las historias clínicas de los pacientes operados de hernia inguinal en la UCMA del $\mathrm{HCH}$ entre julio del 2013 y junio del 2016. Se incluyeron los pacientes con diagnóstico confirmado de hernia inguinal unilateral. Se excluyeron los pacientes que no cumplieron con este criterio.

La técnica quirúrgica fue decisión del cirujano, sea esta en forma abierta (Lichtenstein) o laparoscópica. La técnica de Lichtenstein es un procedimiento que utiliza una malla como prótesis con el fin de reforzar la pared posterior del conducto inguinal, es considerada como la técnica ideal en la cirugía abierta de la hernia inguinal, por ser sencilla y muy baja recidiva, sobre todo en la hernia inguinal unilateral no complicada. En las técnicas laparoscópicas, se utilizaron la técnica transabdominal (TAPP) con apertura del peritoneo 
parietal y colocando la malla en el espacio pre peritoneal, y la técnica extraperitoneal (TEP), con un abordaje por el espacio preperitoneal mediante un trocar balón que llega hasta este espacio bajo visión directa y la colocación de la malla.

La técnica anestésica dependió del tipo de cirugía; en la reparación por técnica abierta la anestesia más usada fue la anestesia local más sedación, por las ventajas que presentaba, su rápida recuperación, confort, tolerancia, mejor función ventilatoria $\mathrm{y}$ disminución del riesgo operatorio; la anestesia regional, fue una alternativa ante la contraindicación, fracaso o negación de la anestesia local. La anestesia general se utilizó en todos los pacientes con reparación laparoscópica. El protocolo de recuperación y control del dolor tuvieron dos fases: la primera post anestesia, en la que se monitorizaron las funciones vitales y la saturación de oxígeno ( $>92 \%$ ) y se trataron las náuseas, vómitos y el dolor postoperatorio; la segunda fase donde se inició la tolerancia oral y se preparó el alta hospitalaria, momento que el paciente con el familiar recibe por escrito las recomendaciones post operatorias, signos de alarma y las instrucciones en casos de complicaciones.

Para las variables continuas se determinaron media y desviación estándar siendo analizados mediante el test de student. En las variables categóricas se determinaron frecuencias y porcentajes, para la comparación se utilizó la prueba de chi cuadrado. Se consideró un nivel de significancia de $\mathrm{p}<0,05$.

El estudio fue aprobado por el Comité Institucional de Ética en Investigación del $\mathrm{HCH}$, y exonerado de revisión por el Comité Institucional de Ética en Investigación-Humanos de la Universidad peruana Cayetano Heredia (UPCH).

\section{RESULTADOS}

Se revisaron 196 historias clínicas de la base de datos del Departamento de Cirugía del $\mathrm{HCH}$ correspondientes a hernioplastías inguinales realizadas en la UCMA entre julio del 2013 y junio del 2016. Dieciocho historias clínicas no fueron encontradas o no coincidían con el procedimiento. Veintiuno fueron descartados por no corresponder a las condiciones del estudio: nueve con hernioplastía bilateral, nueve con hernioplastía crurales, y tres sin evidencia de portar hernia en la región inguinal. No se encontraron registros de la cirugía en tres pacientes, por lo que el número final de historias clínicas incluidas fue 154.
La edad promedio de los pacientes fue $42,95 \pm$ 12,9 años, 101 fueron de sexo masculino, 2/3 tuvieron riesgo quirúrgico ASA I, y 12 tuvieron enfermedades asociadas: 5 con hipertensión arterial, 3 con asma bronquial, 2 con hipotiroidismo y 2 con diabetes mellitus tipo 2 (tabla 1).

De los 154 pacientes, 94 (61\%) se operaron con la técnica abierta, y 60 (39\%) por la vía laparoscópica, de estos 33 (55\%) usaron la técnica TAPP y 27 (45\%) la técnica TEP. Las mujeres fueron programadas con más frecuencia para la técnica laparoscópica $(p=0,037)$. El tiempo operatorio fue mayor en la técnica laparoscópica $(p=0,033)$. No hubo diferencia significativa en las complicaciones anestésicas y quirúrgicas entre cirugía abierta y cirugía laparoscópica (tabla 2).

Los pacientes operados con la técnica abierta (Lichtenstein) recibieron anestesia regional y los operados con las técnicas laparoscópicas recibieron anestesia general. En todos los casos, la recuperación post operatoria y el alta se realizaron de acuerdo al protocolo (3) y el dolor fue tratado con una combinación de antiinflamatorios no esteroideos (AINE) y opioides.

Treinta y cuatro (22\%) pacientes no salieron de alta el mismo día de la cirugía: $26(16,8 \%)$ pacientes se hospitalizaron porque la cirugía terminó muy tarde, hicieron la recuperación dentro del hospital y a pesar de no tener criterios para hospitalización se quedaron hasta el día siguiente por no haber logística para tramitar el alta. Ocho $(5,2 \%)$ pacientes se hospitalizaron por presentar complicaciones: cuatro en la cirugía abierta, dos por punción dural advertida, y dos por hernia de disección difícil y con colocación de drenaje local; y cuatro en la cirugía laparoscópica, dos pacientes operados por técnica TAPP, las dos por taquicardia intraoperatoria; $y$ dos pacientes por técnica

Tabla 1. Características preoperatorias de los pacientes con hernia inguinal unilateral $(\mathrm{n}=154)$.

\begin{tabular}{lcc}
\hline & $\mathbf{n}$ & $\mathbf{\%}$ \\
\hline Sexo & & \\
$\quad$ Masculino & 101 & 65,6 \\
$\quad$ Femenino & 53 & 34,4 \\
Enfermedad asociada & 12 & 7,8 \\
Riesgo Quirúrgico & & \\
$\quad$ ASA I & 104 & 67,5 \\
$\quad$ ASA II & 50 & 32,5 \\
\hline
\end{tabular}


Tabla 2. Resultados según tipo de cirugía.

\begin{tabular}{|c|c|c|c|c|c|}
\hline & \multicolumn{2}{|c|}{$\begin{array}{c}\text { CIRUGIA } \\
\text { ABIERTA } \\
(\mathbf{n}=94)\end{array}$} & \multicolumn{2}{|c|}{$\begin{array}{c}\text { CIRUGIA } \\
\text { LAPAROSCÓPICA } \\
(\mathrm{n}=60)\end{array}$} & \multirow[t]{2}{*}{$\mathbf{p}$} \\
\hline & $\mathbf{n}$ & $\%$ & $\mathbf{n}$ & $\%$ & \\
\hline Edad (años) (media $\pm \mathrm{DE})$ & \multicolumn{2}{|c|}{$39,1 \pm 12,7$} & \multicolumn{2}{|c|}{$42,4 \pm 13,4$} & $0,126^{\&}$ \\
\hline Sexo & & & & & $0,037 *$ \\
\hline Masculino & 68 & 72,3 & 33 & 55,0 & \\
\hline Femenino & 26 & 27,7 & 27 & 45,0 & \\
\hline Enfermedad asociada & 5 & 5,3 & 7 & 11,7 & $0,217 *$ \\
\hline Riesgo Quirúrgico & & & & & $0,223 *$ \\
\hline ASA I & 67 & 71,3 & 37 & 61,7 & \\
\hline ASA II & 27 & 28,7 & 23 & 38,3 & \\
\hline Tiempo operatorio (min) (media \pm DE) & \multicolumn{2}{|c|}{$68,0 \pm 20,7$} & \multicolumn{2}{|c|}{$83,2 \pm 23,5$} & $0,033 \&$ \\
\hline Complicaciones Anestesia & 2 & 2,1 & 3 & 5,0 & $1,000 *$ \\
\hline Complicaciones Cirugía & 2 & 2,1 & 1 & 1,7 & $0,643 *$ \\
\hline
\end{tabular}

\& Prueba t de student; * Chi cuadrado / Prueba exacta de Fisher

TEP, una por antecedente neurológico de convulsiones post-trauma craneano, y una por adherencias del saco herniario que requirió conversión a la técnica abierta. Hubo un caso con la técnica TAPP que se convirtió por presentar una hernia en pantalón, pero el paciente salió de alta dentro de las 8 horas establecidas. No hubo mortalidad en el periodo estudiado.

\section{DISCUSIÓN}

La demanda de pacientes esperando ser operados de enfermedades del aparato digestivo y la pared abdominales es alta, teniendo prioridad en las hospitalizaciones los casos complejos. Las cirugías de menor complejidad, como son las afecciones de la pared abdominal, entre las que se encuentran las hernias umbilicales, inguinales, crurales y las eventraciones, en muchos casos deben de ser resueltas mediante cirugía ambulatoria.

En el 2004 la Agencia Nacional de Salud (NHS) de Inglaterra publicó una guía explicando los 10 cambios de alto impacto con el fin de mejorar los servicios de salud y disminuir la lista de espera para cirugías electivas, con fuerte recomendación de cirugía ambulatoria para la hernia inguinal ${ }^{(8)}$. En el 2013 se aprobó la directiva que permite el funcionamiento de la Unidad de Cirugía Mayor Ambulatoria (UCMA) en el Hospital Cayetano Heredia (HCH) en donde la cirugía de hernia inguinal está entre los procedimientos seleccionados ${ }^{(3)}$.
En este estudio se encontró que las características generales como la edad, sexo, riesgo quirúrgico y enfermedades asociadas no difieren de lo que está reportado en otros trabajos ${ }^{(4,6,9-14)}$.

La selección de la técnica quirúrgica fue decisión del cirujano. El $61 \%$ de pacientes fueron operados por la vía abierta (técnica de Lichtenstein), conocida por ser la más segura y eficaz en la cirugía ambulatoria, como lo señala Sa Ribeiro et al. ${ }^{(14)}$, en una revisión de 454 cirugías de hernia inguinal tipo Linchtenstein con anestesia local, siendo el procedimiento seguro y con aceptación satisfactoria por los pacientes.

La hernioplastia laparoscópica se realizó en 39\% de pacientes, siendo la técnica TAPP la más utilizada. En este estudio se encontró que el tiempo operatorio de la cirugía laparoscópica fue mayor que la cirugía convencional, lo que no debería ser óbice para inhibir la estrategia quirúrgica ambulatoria, debido a los buenos resultados pos operatorios inmediatos que benefician al paciente, como el retorno laboral temprano ${ }^{(15)}$.

Lim et al. ${ }^{(10)}$, evaluaron 706 hernioplastias laparoscópicas ambulatorias tipo TAPP, el tiempo operatorio promedio fue de 41,4 minutos, 3 pacientes se convirtieron a cirugía abierta, $93 \%$ de pacientes salieron de alta en un promedio de 8 horas con $95 \%$ de satisfacción. Reyes et al. (6), presentaron 105 hernias inguinales operadas por la técnica TEP con un promedio de 50 minutos, $5,71 \%$ se convirtieron a cirugía abierta y el $76,2 \%$ fueron en 
régimen ambulatorio. Lau et al. ${ }^{(16)}$, comparó 100 operados con cirugía abierta (Lichtenstein) con 100 pacientes operados con cirugía laparoscópica (TEP) y encontraron diferencias significativas a favor de la técnica TEP en el tiempo operatorio (50 vs 58 minutos) y el dolor postoperatorio. Planells-Roig et al. ${ }^{(17)}$, reportaron menos dolor postoperatorio, menos consumo de analgésicos y recuperación más rápida para las actividades diarias, en los pacientes operados de hernia inguinal de manera ambulatoria con la técnica TEP comparado con la técnica de Lichtenstein. El Grupo Hernia-Surge publicó a inicios del 2018 una guía internacional para el manejo de la hernia inguinal con el objetivo de mejorar los resultados, en especial con las recurrencias y el dolor crónico, recomendando el uso de la laparoscopía en pacientes seleccionados para cirugía ambulatoria ${ }^{(18)}$.

LaAsociación Internacional de Cirugía Ambulatoria (IAAS) en su sección de recomendaciones, coloca algunos indicadores clínicos como parámetros de calidad de la cirugía ambulatoria ${ }^{(19)}$. El indicador 3 se refiere a las hospitalizaciones no planificadas (HNP) que pueden ser causadas por razones quirúrgicas, razones clínicas/anestésicas y por razones sociales/ administrativas, evitando que el paciente se vaya de alta el mismo día de la cirugía.

En general, el éxito de una UCMA requiere un cambio en los paradigmas donde las ventajas de disminuir la espera para las cirugías de mediana y baja complejidad, bajar los costos, disminuir las tasas de infección intrahospitalaria y disponer de camas para cirugía de mayor complejidad, se sobrepongan a las dificultades operacionales y administrativas de estos programas ${ }^{(20-22)}$.

La cirugía ambulatoria de hernia inguinal ha crecido en todo el mundo, se realizan con cualquier modalidad de anestesia y técnica quirúrgica, no demandan cama de hospitalización, requieren de un periodo de monitorización y recuperación postoperatoria que no excede de 8 horas ${ }^{(4)}$. Está asociada a cuidados de alta calidad, con el objetivo de que el paciente se beneficie de un alta temprana y regrese a sus actividades lo más pronto posible, con mínimos eventos adversos (20). El éxito de la cirugía ambulatoria depende de la integración del equipo multiprofesional, de protocolos rigurosos de selección, de sistematización de las conductas, del acompañamiento del paciente por los familiares y de la seguridad que entendieron las orientaciones médicas después del alta ${ }^{(20)}$.
Según la Sociedad Europea de Hernia, la cirugía de la hernia inguinal unilateral con el sistema de UCMA está reconocida en las guías médicas con nivel de evidencia 1 , y grado A de recomendación ${ }^{(22)}$.

Hay limitaciones relacionadas al diseño transversal retrospectivo para determinar el efecto de la cirugía de hernia sobre las recidivas; sin embargo, los cirujanos de la UCMA, siendo parte del servicio de cirugía general, usaron las mismas técnicas de los pacientes hospitalizados. El estudio solo contempló las complicaciones que sucedieron durante la cirugía, $\mathrm{y}$ en el periodo que permanecieron en la UCMA, quedando por evaluar el periodo que el paciente pasa en su domicilio después del alta, hasta las primeras consultas de control post operatorio. Asimismo, deberían hacerse análisis de costo beneficio de la cirugía laparoscópica en una UCMA de un hospital público.

Se puede concluir que la cirugía de reparación de la hernia inguinal unilateral en adultos en la UCMA del $\mathrm{HCH}$, realizada por la técnica abierta o laparoscópica, siguiendo estrictamente el protocolo, presenta pocos eventos adversos durante la estancia, y permite, con seguridad, el alta del paciente el mismo día del procedimiento.

\section{Declaración de financiamiento y de conflictos de interés:}

Los autores declaran mediante juramento, que el estudio fue financiado por los autores, y declaran no tener conflictos de intereses con persona o institución alguna, ni de orden económico, ni institucional, ni laboral, ni personal.

\section{Contribución de autoría:}

REVG: Diseño del estudio, revisión de historias clínicas, recolección de datos, análisis e interpretación de los resultados, redacción, referencias bibliográficas, aprobación de la versión final a ser publicada. MACY: diseño del estudio, referencias bibliográficas, revisión crítica del artículo, aprobación de la versión final a ser publicada.

\section{Correspondencia:}

Roberto Vigil Guerrero.

Av Principal 676 (1101) Surquillo, Lima 34, Perú.

Correo electrónico: rvg444@hotmail.com

Celular: +55 92991987609 


\section{REFERENCIAS BIBLIOGRÁFICAS}

1. Ministerio de Salud del Perú. Guía práctica clínica de Hernia inguinal del Departamento de Cirugía del Hospital Nacional Cayetano Heredia. Lima: MINSA; 2013. (Citado 26 de abril de 2017). Disponible en: http://www.hospitalcayetano.gob.pe/transparencia/ images/stories/resoluciones/RD/RD2013/ rd 510 2013.pdf

2. Ministerio de Salud del Perú. Directiva del proceso de atención para el usuario externo de las cirugías electivas del Hospital Nacional Cayetano Heredia. Lima: MINSA; 2013. (Citado 26 de abril de 2017). Disponible en: http://www.hospitalcayetano.gob.pe/ transparencia/images/stories/resoluciones/RD/ RD2013/rd102_2013.pdf

3. Scarfe A, Duncan J, Ma N, et al. Day case hernia repair: weak evidence or practice gap? ANZ J Surg. 2018 Jun; 88(6):547-553.

4. Saia M, Mantoan D, Buja A, et al. Increased rate of day surgery use for inguinal and femoral hernia repair in a decade of hospital admissions in the Veneto Region (north-east Italy): a record linkage study. BMC Health Serv Res. Sep 12 2013; 13:349.

5. Fernández-Ordóñez M, Tenías JM, Picazo-Yeste J. Anestesia subaracnoidea y anestesia general en el tratamiento quirúrgico de la hernia inguinal en pacientes ambulatorios. Análisis comparativo de coste-efectividad. Rev Esp Anestesiol Reanim. 2014; 61(5):254-61.

6. Reyes ML, Naranjo JR, Domínguez A, et al. Reparación herniaria mediante abordaje laparoscópico totalmente extraperitoneal en una Unidad de Cirugía Mayor Ambulatoria: ¿Avanzamos hacia el futuro? Rev Port Cir. 2014;(30):13-8.

7. Martinez-Ramos C. Cirugía Mayor Ambulatoria. Concepto y Evolución. REduca. 2009;1(1): 294-304. (Citado 26 de abril de 2017). Disponible en: http:// www.revistareduca.es/index.php/reduca/article/ view/61

8. NHS Modernisation Agency. 10 High Impact Changes for Service Improvement and Delivery. Leicester: NHS Modernisation Agency; 2004. (Citado 26 abril 2017). Disponible en: https://www. england.nhs.uk/improvement-hub/wp-content/ uploads/sites/44/2017/11/10-High-Impact-Changes. pdf

9. McCloud JM, Evans DS. Day-case laparoscopic hernia repair in a single unit. Surg Endosc. 2003;17(3):4913.

10. Lim M, O’Boyle CJ, Royston CMS, Sedman PC. Day case laparoscopic herniorraphy. A NICE procedure with a long learning curve. Surg Endosc. 2006; 20(9):1453-9.

11. Lima E, Goldenberg A, Juca M. Immediate results of inguinal hernia repair with local anesthesia associated with sedation. Acta Cir Bras. 2003;18(5):478-84.

12. Peres M, Nieri T, Barcelos-Neto H, Andreollo N. A técnica de Lichtenstein nas hérnias inguinais primárias e recidivadas - cirurgia ambulatorial em hospital universitário ABCD Arq Bras Cir Dig São Paulo. 2007; 20(4):221-4.

13. Dhumale R, Tisdale J, Barwell N. Over a thousand ambulatory hernia repairs in a primary care setting. Ann R Coll Surg Engl. 2010; 92(2):127-30.

14. Ribeiro F, Padron F. Herniorrafia inguinal com anestesia local em regime ambulatorial. Rev Col Bras Cir. 2010; 37(6):397-402.

15. Wall ML, Cherian T, Lotz JC. Laparoscopic hernia repair--the best option? Acta Chir Belg. 2008 MarApr; 108(2):186-91.

16. Lau H, Patil NG, Yuen WK. Day-case endoscopic totally extraperitoneal inguinal hernioplasty versus open Lichtenstein hernioplasty for unilateral primary inguinal hernia in males: a randomized trial. Surg Endosc. 2006; 20(1):76-81.

17. Roig MP, Bertomeu CA, Delgado MC, et al. Dolor percibido, consumo de analgésicos y recuperación de las actividades de la vida diaria en pacientes sometidos a hernioplastia inguinal ambulatoria laparoscópica tipo TEP versus hernioplastia Lichtenstein en régimen ambulatorio. Cir Esp. 2011 Oct; 89(8):524-31.

18. HerniaSurge Group. International guidelines for groin hernia management. Hernia. 2018 Feb; 22(1):1-165. International Association for Ambulatory Surgery. Clinical Indicators for Ambulatory Surgery. Londres: International Association for Ambulatory Surgery; 2015. (Citado 26 de abril de 2017). Disponible en: $\quad$ http://www.iaas-med.com/index.php/iaasrecommendations/clinical-indicators

19. Hammond CB. Care of patients undergoing day case inguinal hernia repair. Nurs Stand. 2014; 28(31):539.

20. Bomtemps G. Recomendations organisationnelles de la chirurgie ambulatorie. Presse Med. 2014; 43:309318.

21. Miserez M, Peeters E, Aufenacker T, et al. Update with level 1 studies of the European Hernia Society guidelines on the treatment of inguinal hernia in adult patients. Hernia. Apr 2014; 18(2):151-63.

Recibido: 11/11/2020

Aceptado: 23/09/2021 